\title{
Hematological, parasitological and biochemical parameters in sheep during the peripartum period
}

\author{
Parámetros hematológicos, parasitológicos y bioquímicos en ovejas durante el periparto \\ Parâmetros hematológicos, parasitológicos e bioquímicos de ovelhas durante o periparto
}

\author{
Caroline M G David ${ }^{1}$ D) Ricardo L D Costa $^{1 *}$ iD; Guadalupe A E Parren ${ }^{1}$ (D); Miguel A S Rua ${ }^{1}$ iD; Ellen C P Nordi ${ }^{1}$ iD; \\ Cláudia C P Paz ${ }^{2}$ D ; Celia R Quirino 3 iD; Rogério S Figueiredo4 (D); Elizabeth Bohland ${ }^{4}$.
}

\author{
${ }^{1}$ Centro de Pesquisa e Desenvolvimento em Zootecnia Diversificada/Instituto de Zootecnia/Apta. Rua Heitor Penteado, 56, Nova Odessa, São \\ Paulo, Brazil, 13460-000. \\ ${ }^{2}$ Centro APTA de Bovinos de Corte-IZ/APTA, Sertãozinho-SP, Brazil; CNPq Productivity Fellowship. \\ ${ }^{3}$ Universidade Estadual Norte Fluminense - Uenf, Campos dos Goytacazes, Rio de Janeiro, Brazil; CNPq Productivity Fellowship. \\ ${ }^{4}$ Universidade de Santo Amaro - UNISA, São Paulo, Brazil; + Unisa Fellowhip.
}

\section{To cite this article:}

David CMG, Costa RLD, Parren GAE, Rua MAS, Nordi ECP, Paz CCP, Quirino CR, Figueiredo RS, Bohland E.

Hematological, parasitological and biochemical parameters of sheep during peripartum. Rev Colomb Cienc Pecu 2020; 33(2):

81-95. DOI: https://doi.org/10.17533/udea.rccp.v33n1a04

\begin{abstract}
Background: Pregnancy in all animal species requires special care and attention. During this period there is great variation in hematological and biochemical parameters. Many times these parameters are misinterpreted as being obtained from nonpregnant animals with unknown parasite status. Objective: To characterize the hematological, biochemical, and parasitological values within the peripartum period of Santa Ines sheep naturally infected. Methods: For this purpose, 45 ewes were used, with an average body weight of $50 \mathrm{~kg}$. Data were collected during pregnancy, birth and, lactation. Measurements included body weight, body condition score (BCS), evaluation of conjunctive staining, and collection of blood and feces. Results: All variables changed throughout physiological stages. Weight and BCS were different between periods ( $\mathrm{p}<0.05)$. Fecal egg counts (FEC) were higher in the peripartum period, with a predominance of Haemonchus ssp, 92.85\%. Mean hematological and biochemical values varied throughout the study, $(\mathrm{p}>0.05)$. Conclusion: The influence of peripartum on the parameters studied should be considered and its use as a reference value. Regarding the physiological variations observed during peripartum, proper animal management could help minimizing the negative effects of parasitic infections on productivity.
\end{abstract}

Keywords: ewes; gestation; Haemonchus contortus; hematology; laboratory diagnosis; nematodes; Ovis aries; parasites; peripartum; Santa Ines; sheep.

Received: March 12, 2018; accepted: August 22, 2019

*Corresponding author: Rua Heitor Penteado, 56, Nova Odessa, São Paulo, Brazil, 13460-000. Tel.: (55) 19 981186180. E-mail: rldcosta@iz.sp.gov.br 


\section{Resumen}

Antecedentes: La preñez en todas las especies animales requiere atención y cuidados especiales. En ese período existe gran variación en los parámetros hematológicos y bioquímicos. Muchas veces estos parámetros son interpretados erróneamente por ser obtenidos de animales no gestantes y con estatus parasitario desconocido. Objetivo: Caracterizar los valores hematológicos, bioquímicos y parasitológicos durante el periparto en ovejas Santa Inés infectadas naturalmente. Métodos: Se utilizaron 45 ovejas, con un peso corporal promedio de $50 \mathrm{~kg}$. la recolección de muestras se realizó durante la gestación, el parto y la lactancia. Se evaluó el peso, la condición corporal, la tinción conjuntiva, y se recolectaron muestras de sangre y heces. Resultados: Todas las variables mostraron variaciones a lo largo de las etapas fisiológicas. La puntuación de peso y condición corporal (BCS) mostró diferencia entre períodos $(\mathrm{p}<0,05)$. Los recuentos de huevos fecales (FEC) tuvieron mayores promedios en el periparto, con predominio de Haemonchus ssp, 92,85\%. Los valores medios hematológicos y bioquímicos variaron a lo largo del estudio $(\mathrm{p}<0,05)$. Conclusiones: Se recomienda considerar la influencia del periparto en los parámetros estudiados y su uso como valor de referencia. Con respecto a las variaciones fisiológicas observadas durante el periparto, el manejo adecuado de los animales podría ayudar a minimizar los efectos negativos de las infecciones parasitarias sobre la productividad.

Palabras clave: diagnóstico de laboratorio; gestación; Haemonchus contortus; hematología; periparto; nematodos; ovejas; Ovis aries; parasitos; Santa Inés.

\section{Resumo}

Antecedentes: A prenhez em todas as espécies requer atenção e cuidados especiais. Neste período há grande variação dos parâmetros hematológicos e bioquímicos dos animais. Muitas vezes estes parâmetros são mal interpretados na realização de diagnósticos por serem baseados em parâmetros de animais saudáveis não gestantes, desconsiderando também a infecção parasitária. Objetivo: Caracterizar os valores hematológicos, bioquímicos e parasitológicos durante o periparto de ovelhas da raça Santa Inês naturalmente infectadas. Métodos: Foram utilizadas quarenta e cinco ovelhas, com peso corporal médio de $50 \mathrm{~kg}$, os animais foram acompanhados durante a prenhez, parto e lactação. As análises compreendiam: pesagem, condição corporal, Famacha $($, sangue e fezes. Resultados: Todas as variáveis apresentaram variações concomitantemente ao avanço da gestação, parto e lactação. O peso (PS) e o escore da condição corporal (ECC) apresentaram diferença estatística entre os períodos ( $\mathrm{p}>0,05)$. As contagens de ovos por grama de fezes (FEC) apresentaram médias mais elevadas no periparto. Os valores hematológicos e bioquímicos também variaram $(\mathrm{p}>0,05)$. Conclusão: Devido ao resultado, sugere-se considerar a influência de periparto sobre os parâmetros estudados e a sua utilização como um valor de referência. Tendo em vista as variações fisiológicas devido o período do periparto e as infecções parasitárias o manejo dos animais deve ter como objetivo minimizar os impactos negativos na produção animal.

Palavras-chave: diagnóstico laboratorial; fenômeno do periparto; gestação; Haemonchus contortus; hematologia; nematoides; ovelhas; Ovis $\underline{\text { aries; }}$ parasitos; Santa Inês. 


\section{Introduction}

Sheep breeding is exploited on all continents, with wide dissemination mainly due to its ability to adapt to different climates, terrain and vegetation. Mutton production experienced 27\% growth in Brazil over the last 20 years, despite the reduced demand for wool (MAPA, 2013).

The increase in mutton consumption has required greater production efficiency. Therefore, it is necessary to improve the management of herds through detailed characterization of breeds, breeding systems and critical phases, taking into account the different production regions. Nutritional and health problems are the main cause of the limited efficiency of ruminant production (Hernandez et al., 2013). During the peripartum period, depression of the immune system is directly related to the physiological state of the animal (Silva et al., 2003 to Nieto et al., 2003) due to hormonal changes that occur near lambing and during lactation (Gennari et al., 2002;.Aya et al., 2008). These changes are called "the peripartum phenomenon" (Amarante et al., 2004; Rocha et al., 2004).

Nutrients and energy requirements increase during pre-natal and early lactation, concomitant with a reduction in dry matter intake due to rumen compression by the fetus and production of hormones involved in pregnancy (Rodrigues et al., 2006). This nutritional challenge is also associated with increased susceptibility to gastrointestinal parasites. An increase in the number of nematode eggs per gram of feces in sheep during peripartum can occur due to inadequate supply of dietary protein (Donaldson et al., 1998 and 2001; Houdijk et al., 2001).

Although a number of studies have investigated the association between nutrition and gastrointestinal infections in sheep (Hoste et al., 2005) few have focused on the potential variations of hematological, biochemical and parasitological parameters associated with peripartum, a critical stage for productive and reproductive efficiency. This knowledge is necessary to improve management practices, particularly because of the large heterogeneity of values within this species due to differences of breed, gender, age, nutrition, management systems (Kerr, 2003) and physiological status (Cardoso et al., 2010).

In this study, we characterized the hematological, biochemical and parasitological values in the peripartum period in Santa Ines sheep, the main native breed used in Brazil and one of great interest in other South American countries.

\section{Materials and Methods}

\section{Ethical Considerations}

This study was conducted and approved by the ethics committee for animal research at Instituto de Zootecnia (finding no. 142/2011).

\section{Local}

This studywas conducted atCentro dePesquisa e Desenvolvimento de Zootecnia Diversificada, at Instituto de Zootecnia, of Agência Paulista de Tecnologia dos Agronegócios (APTA), da Secretaria de Agricultura e Abastecimento, located in Nova Odessa, São Paulo state $\left(22^{\circ} 42^{\prime}\right.$ $\mathrm{S}$ and $\left.47^{\circ} 18^{\prime} \mathrm{W}\right)$.

According to the Köppen classification, the climate in the Nova Odessa region is CWa, characterized as tropical with relatively dry and hot summers and rainy winters. Average rainfall is $1,317 \mathrm{~mm}$ per year and the temperature is between $10^{\circ} \mathrm{C}$ and $35^{\circ} \mathrm{C}$, with an average of $26^{\circ} \mathrm{C}$. The average annual relative humidity is approximately $75 \%$.

\section{Animals and management}

A total of 70 Santa Ines ewes kept under a natural breeding system were tested for pregnancy by ultrasound. Of these, we used 45 sheep diagnosed with close gestational age and $50 \mathrm{~kg}$ average body weight. Sheep were kept under rotational grazing on Panicum maximum Jacq. cv. Aruana and cv. Tanzania pastures, with ad libitum access to water and mineral mixture. During late pregnancy, sheep were confined in 
paddocks and supplemented with corn silage $\mathrm{ad}$ libitum and $0.300 \mathrm{~kg}$ concentrate $(16 \%$ crude protein). They remained in the paddocks until the lambs were weaned at 3 months of age. Feed samples were taken every 15 days and frozen at $-20{ }^{\circ} \mathrm{C}$ for later analysis.

\section{Sample collection and evaluation of animals}

Blood and stool samples were collected every 14 days during five months, for a total of 10 collections. At this time, sheep were also weighed and evaluated for body condition score and conjunctival staining. Blood samples were also collected for hematological and biochemical analyses on the day of lambing. Body condition score was determined by palpation of the lower back (Sanudo and Sierra, 1986) using a halfpoint scoring (Pugh, 2004). Parasitological and hematological analyses were performed at the Laboratory for Fecal Analysis and Laboratory for Clinical Analysis, respectively, of Instituto de Zootecnia de Nova Odessa/APTA.

\section{Parasitological examinations}

Stool samples were collected directly from the rectum for egg counting (eggs per gram of feces; EPG) according to the modified technique by Gordon and Whitlock (1939). Fecal culture of infective larvae was conducted for identification of parasite genra (Roberts and O’Sullivan, 1950).

\section{Hematological analysis}

Blood samples from all animals were collected by venipuncture of the jugular vein in Vacutainer tubes $(5 \mathrm{ml})$ containing ethylenediaminetetraacetic acid (EDTA). Packed cell volume analysis (PVC) was conducted after microhematocrit centrifugation. Hemoglobin $(\mathrm{Hb})$ concentration was measured by the cyanmethemoglobin method using a semi-automatic biochemical analyzer (Bio $200 \mathrm{~L}$ Bioplus) using commercial kits for hemoglobin determination (Labtest). Total plasma protein (TPP) was determined by the refraction technique. Total leukocyte count (Tot Leuk) and erythrocyte count (RBC) were determined manually in a Neubauer hemocytometer (Schalm and Carroll, 1986). Tests identified blood smears and differential count of segmented neutrophils (Seg Neut), lymphocytes (Lymph), eosinophils (Eosin), monocytes (Mono), basophils (Baso) and rods (Bast) (Schalm and Carroll, 1986). No rods were observed, so this parameter was removed from the statistical analysis.

\section{Biochemical analysis}

The blood samples were subjected to the following biochemical analyses: globulin, albumin, serum protein (Ser $\mathrm{Pt}$ ), urea, creatinine, total bilirubin (BT), direct bilirubin (BD), indirect bilirubin (BI), glucose (Gluco), cholesterol, AST (AST), calcium, iron (Fe), and phosphorus. The tests were performed in a Bio 200L semiautomatic biochemical analyzer (Bioplus, São Paulo, Brazil) using commercial kits (Labtest, Lagoa Santa, Minas Gerais, Brazil).

\section{Statistical analysis}

The assumption of normal distribution of all variables was verified. The variables fibrinogen, total leukocytes, segmented neutrophils, lymphocytes, eosinophils, monocytes, trichostrongylid, Strongyloides spp, Moniezia spp and Eimeria spp which did not meet the assumption of normality were transformed. The data were transformed for the FAMACHA and fibrinogen variables by raising to the -0.2 power. Square roots were used for total leukocytes and erythrocytes, and the other non-normal variables were $\log 10$ transformed. The means are presented in the original scale.

Analysis of variance (ANOVA) was conducted using the PROC GLM routine of SAS for all variables (processed or not) considering repeated measures. The model and analysis considered the effect of weight as a covariate in addition to the effects of treatment and time and their interaction. The means were compared by the Tukey test considering 5\% probability for Type I error. 


\section{Results}

\section{Feeding and performance}

Sheep ate a daily average of $1.85 \mathrm{~kg}$ dry matter and $177 \mathrm{~g}$ crude protein at the beginning of the experiment, with consumption decreasing in the peripartum month (September) and increasing linearly until the end of lactation (November), when it reached $2 \mathrm{~kg}$ dry matter and $200 \mathrm{~g}$ crude protein (Figure 1).

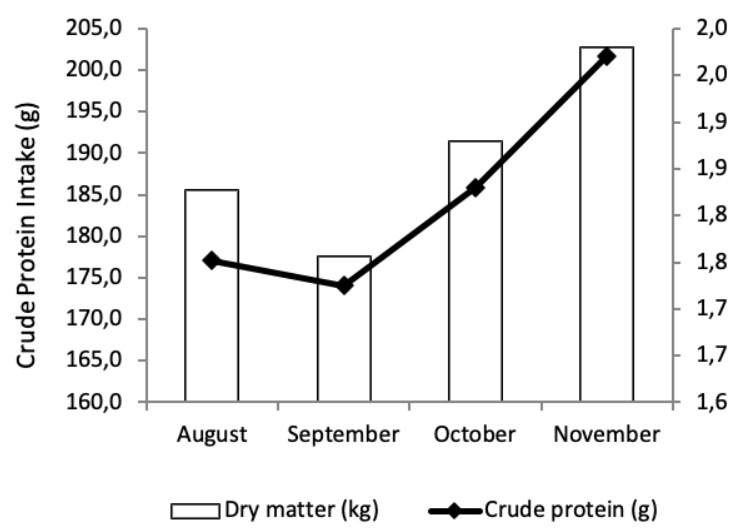

Figure 1. Dry matter and crude protein intake of Santa Ines ewes.
Body weight increased significantly from the beginning of the study until lambing, when it was the highest. Subsequently, there was a sharp and significant drop in weight from day 1-13 after birth, when body weight returned to a value similar to that observed $>30$ days before birth. A further reduction in weight was also observed from days 14-27 (lactation), after which it remained constant. The analysis of BCS showed a significant reduction associated with the peripartum period, which was particularly accentuated during the peak of lactation (up to 83 days after birth). From day 84 onwards, BCS was not different from that during pregnancy (Table 1).

\section{Parasitological analysis}

Table 2 shows the results of the endoparasite analysis. The mean FAMACHA score varied from 1.2 (1-13 days) to 1.7 (<-30 days), with no difference between periods $(p>0.05)$. Trichostrongylid FEC was, however, significantly higher during gestation and peripartum than during lactation, with a particularly sharp reduction in FEC towards the end of the latter period.

Table 1. Least square means of body weight and body condition score (BCS) of Santa Ines ewes.

\begin{tabular}{|c|c|c|c|}
\hline & Days & BW & BCS \\
\hline \multirow[t]{2}{*}{ Pregnancy } & $<-30$ & $48.1^{\mathrm{a}}$ & $2.9^{\mathrm{a}}$ \\
\hline & $>14-30$ & $52.3^{\mathrm{b}}$ & $2.8^{\mathrm{ab}}$ \\
\hline \multirow[t]{3}{*}{ Peripartum } & $>1-13$ & $53.8^{\mathrm{c}}$ & $2.6^{\mathrm{bcd}}$ \\
\hline & Partum & . & . \\
\hline & $1-13$ & $47.7^{\mathrm{a}}$ & $2.6^{\text {be }}$ \\
\hline \multirow[t]{5}{*}{ Lactation } & $\geq 14-27$ & $45.6^{\mathrm{d}}$ & $2.5^{\mathrm{ce}}$ \\
\hline & $\geq 28-55$ & $44.7^{\mathrm{d}}$ & $2.5^{\mathrm{ce}}$ \\
\hline & $\geq 56-83$ & $45.5^{\mathrm{d}}$ & $2.5^{\text {be }}$ \\
\hline & $>84$ & $44.9^{d}$ & $2.6^{\text {ade }}$ \\
\hline & $\mathrm{SE}$ & \pm 1.1 & \pm 0.1 \\
\hline
\end{tabular}

Different superscript letters $\left({ }^{a}, b, c, d, e\right)$ within the same column indicate significant differences at $\mathrm{p}<0.05$. 
Table 2. Least square means of FAMACHA ${ }^{\odot}( \pm$ standard error) and parasitological variables of Santa Ines ewes ( \pm standard error).

\begin{tabular}{ccccccc}
\hline & & FAMACHA $^{*}$ & Tricho & Strong & Moni SD & Eime SD \\
\hline \multirow{3}{*}{ Pregnancy } & $>30$ & $1.7 \pm 0.1$ & $2885 \pm 290^{\mathrm{a}}$ & $11.8 \pm 90.2^{\mathrm{ab}}$ & $195.6 \pm 297.50^{\mathrm{a}}$ & $34.2 \pm 130.3^{\mathrm{a}}$ \\
& $>14-30$ & $1.5 \pm 0.1$ & $3296 \pm 32^{\mathrm{a}}$ & $35.7 \pm 104.0^{\mathrm{ab}}$ & $228.6 \pm 315.68^{\mathrm{a}}$ & $14.3 \pm 78.3^{\mathrm{a}}$ \\
& $>1-13$ & $1.4 \pm 0.1$ & $2659 \pm 315^{\mathrm{ab}}$ & $180.6 \pm 101.6^{\mathrm{c}}$ & $34.1 \pm 120.45^{\mathrm{b}}$ & $4.5 \pm 14.5^{\mathrm{a}}$ \\
\multirow{3}{*}{ Peripartum } & & & & & \\
& Day 0 & $1.1 \pm 0.4$ & $2324 \pm 1119^{\mathrm{ac}}$ & $836.5 \pm 388.8^{\mathrm{acde}}$ & $0 \pm 0.0^{\mathrm{b}}$ & $0 \pm 0^{\mathrm{ab}}$ \\
& $1-13$ & $1.2 \pm 0.1$ & $2457 \pm 318 \mathrm{a}^{\mathrm{d}}$ & $758.1 \pm 102.8^{\mathrm{d}}$ & $0 \pm 0.0^{\mathrm{b}}$ & $25.6 \pm 152.5^{\mathrm{a}}$ \\
& $\geq 14-27$ & $1.4 \pm 0.1$ & $1923 \pm 318^{\mathrm{bcd}}$ & $436.5 \pm 102.8^{\mathrm{d}}$ & $0 \pm 0.0^{\mathrm{b}}$ & $31.4 \pm 87.3^{\mathrm{a}}$ \\
& & & & & \\
& & & & & \\
& $>28-55$ & $1.3 \pm 0.1$ & $1128 \pm 243^{\mathrm{c}}$ & $340.6 \pm 72.0^{\mathrm{e}}$ & $2.2 \pm 12.8^{\mathrm{b}}$ & $544.4 \pm 3665^{\mathrm{b}}$ \\
& $>56-83$ & $1.3 \pm 0.1$ & $483 \pm 250^{\mathrm{e}}$ & $72.1 \pm 74.9^{\mathrm{c}}$ & $42.8 \pm 336.1^{\mathrm{b}}$ & $681.1 \pm 1842^{\mathrm{c}}$ \\
& $>84$ & $1.4 \pm 0.1$ & $273 \pm 375^{\mathrm{f}}$ & $3.8 \pm 123.2^{\mathrm{b}}$ & $18.3 \pm 74.8^{\mathrm{b}}$ & $78.3 \pm 133.1 \mathrm{a}^{\mathrm{b}}$ \\
\hline
\end{tabular}

*In those cases where ER, SE were used. Otherwise, different superscript letters (a, b, c, d, e) within the same column indicate significant differences at $\mathrm{p}<0.05$ (Tukey-Kramer). Thrichostrongylides (Tricho), Strongyloides spp (Strong), Moniezia spp (Moni), Eimeria spp (Eime).

Unexpectedly, Trichostrongylid FEC was not significantly higher on the lambing date. Strongyloides spp. FEC increased from gestation to birth, with accentuated increase on the birth date, and subsequent reduction afterwards. The Moniezia spp. count showed the highest values in the first two gestational periods, while Eimeria counts had the lowest values in the first six periods (pregnancy, peripartum and lactation up to 27 days postpartum), differing from later lactation periods.

The coproculture results showed average counts of Trichostrongylidae, which is the parasite genus of greatest interest, with predominance of Haemonchus ssp (92.85\%).

\section{Hematological profile}

The hematological variables showed significant variations across the gestational period (Table 3 ). As expected, there was a gradual increase in packed cell volume over the course of the study, and a decrease in mean corpuscular volume (MVC). The lowest values of hemoglobin concentration ( $\mathrm{Hb})$, total plasma protein (TPP), and mean corpuscular hemoglobin concentration (MCHC) were observed near the birth date.
This date was also associated with the highest values of leucocytes, neutrophils and lymphocytes (Table 4). Eosinophils and monocytes were, conversely, particularly low at birth.

\section{Blood biochemistry}

All blood biochemistry analyses, regarding metabolites, enzymes (Tables 5 and 6) and minerals (Table 7), were performed in a Bio 200L semiautomatic biochemical analyzer (Bioplus, São Paulo, Brazil), with Labtest commercial kits (Labtest, Lagoa Santa, Minas Gerais, Brazil). Blood levels of metabolites, globulin, albumin, total serum protein, urea, creatinine, glucose (Table 5) total bilirubin, direct bilirubin, indirect bilirubin, cholesterol and AST differed significantly $(p<0.05)$ with the different physiological conditions (Table 6). The globulin values ranged from $3.88(\geq 14-27$ days) to 4.85 ( $>84$ days), with a significant increase over the course of lactation. The values of albumin and serum protein were significantly lower in the birth date, although similar to the average associated with day 84 onwards. The values of urea (Tab 5) decreased from gestation to peripartum, subsequently increasing over the lactation period. There were no clear trends in the variation of creatinine values. 
Sheep had lower levels of total, direct and indirect bilirubin on lambing day $(\mathrm{p}>0.05)$ compared to all other periods, except for the direct bilirubin, which was similar to that of the period $>84$ days postpartum (Table 6 ).

Cholesterol and AST had constant variations during all periods, with respective amplitudes of
$48.2 \pm 1.8$ (1-13 days) to $60.8 \pm 1.8(>1-13)$ and $82.6 \pm 3.7(\geq 14-27)$ to $111.0 \pm 4.4(>84)$.

The serum levels of calcium, phosphorus and iron showed significant differences $(p<0.0001)$ between the periods of pregnancy, birth and lactation. The mean changes in calcium, phosphorus and iron were independent of the physiological condition.

Table 3. Mean ( \pm standard error) of hematological variables of Santa Ines ewes, according to physiological stage.

\begin{tabular}{|c|c|c|c|c|c|c|c|c|}
\hline & & $\begin{array}{c}\text { RBC } \\
(106 / \mu \mathrm{L})\end{array}$ & PCV (\%) & Hb (g/dL) & TPP(g/dL) & MCV (fL) & МСH & MCHC (\%) \\
\hline \multirow[t]{3}{*}{ Pregnancy } & $>30$ & $7.18 \pm 0.2^{\mathrm{a}}$ & $27.41 \pm 0.60^{\mathrm{ab}}$ & $10.01 \pm 0.2^{\mathrm{ab}}$ & $6.7 \pm 0.09^{\mathrm{a}}$ & $39.96 \pm 0.65^{\mathrm{a}}$ & $3.57 \pm 0.059^{\mathrm{a}}$ & $35.73 \pm 0.59^{\mathrm{a}}$ \\
\hline & $>14-30$ & $7.51 \pm 0.2^{\mathrm{ab}}$ & $27.06 \pm 0.59^{\mathrm{ad}}$ & $10.17 \pm 0.3^{\mathrm{acd}}$ & $6.5 \pm 0.08^{\mathrm{bc}}$ & $36.71 \pm 0.72^{b}$ & $3.82 \pm 0.066^{\mathrm{bc}}$ & $38.23 \pm 0.66^{\mathrm{bd}}$ \\
\hline & $>1-13$ & $7.86 \pm 0.2^{\mathrm{bc}}$ & $28.03 \pm 0.59^{b}$ & $10.57 \pm 0.3^{\text {ade }}$ & $6.5 \pm 0.09^{\mathrm{ab}}$ & $36.09 \pm 0.73^{b}$ & $3.78 \pm 0.067^{b c}$ & $37.81 \pm 0.67^{\mathrm{bd}}$ \\
\hline \multirow[t]{3}{*}{ Peripartum } & Dia 0 & $7.86 \pm 0.2^{\mathrm{cd}}$ & $28.88 \pm 0.59^{c}$ & $10.00 \pm 0.3^{\mathrm{abc}}$ & $6.4 \pm 0.09^{\mathrm{b}}$ & $36.21 \pm 0.72^{\mathrm{b}}$ & $3.47 \pm 0.068^{\mathrm{a}}$ & $34.73 \pm 0.68^{\mathrm{a}}$ \\
\hline & $1-13$ & $8.39 \pm 0.2^{\mathrm{cd}}$ & $28.25 \pm 0.50 b^{c}$ & $10.71 \pm 0.3^{\mathrm{de}}$ & $6.6 \pm 0.09^{\mathrm{a}}$ & $33.87 \pm 0.73^{\mathrm{c}}$ & $3.85 \pm 0.068^{\mathrm{bc}}$ & $38.54 \pm 0.68^{\text {bd }}$ \\
\hline & $\geq 14-27$ & $8.13 \pm 0.2^{\text {ce }}$ & $26.56 \pm 0.59^{\mathrm{a}}$ & $10.10 \pm 0.3^{\mathrm{ac}}$ & $6.7 \pm 0.09^{\mathrm{a}}$ & $32.95 \pm 0.72^{\mathrm{cd}}$ & $3.78 \pm 0.067^{\mathrm{bc}}$ & $37.78 \pm 0.67^{\mathrm{b}}$ \\
\hline \multirow[t]{3}{*}{ Lactation } & $>28-55$ & $8.41 \pm 0.2^{\mathrm{de}}$ & $26.83 \pm 0.55^{\mathrm{a}}$ & $10.00 \pm 0.2^{\mathrm{bc}}$ & $6.7 \pm 0.08^{\mathrm{a}}$ & $31.98 \pm 0.54^{\mathrm{d}}$ & $3.75 \pm 0.048^{b}$ & $37.55 \pm 0.48^{\mathrm{b}}$ \\
\hline & $>56-83$ & $9.14 \pm 0.2^{\mathrm{f}}$ & $27.46 \pm 0.56^{\mathrm{ab}}$ & $11.55 \pm 0.2^{\mathrm{ef}}$ & $6.8 \pm 0.08^{\mathrm{a}}$ & $30.56 \pm 0.55^{\mathrm{e}}$ & $4.26 \pm 0.049^{\mathrm{d}}$ & $42.61 \pm 0.49^{c}$ \\
\hline & $>84$ & $9.04 \pm 0.3^{f}$ & $28.23 \pm 0.65^{\text {bcd }}$ & $11.06 \pm 0.3^{\mathrm{e}}$ & $6.7 \pm 0.10^{\mathrm{a}}$ & $31.40 \pm 0.87 \mathrm{~d}^{\mathrm{e}}$ & $3.98 \pm 0.080^{\mathrm{c}}$ & $39.83 \pm 0.80^{\mathrm{d}}$ \\
\hline
\end{tabular}

Average followed by different superscript letters $(\mathrm{a}, \mathrm{b}, \mathrm{c}, \mathrm{d}, \mathrm{e})$ within the same column differ by the Tukey's test $(\mathrm{p}<0.05)$. (RBC) erythrocytes, (PCV) packed cell volume, (Hb) hemoglobin concentration, (TPP) total plasm protein, (MCV) mean corpuscular volume, $(\mathrm{MCH})$ mean corpuscular hemoglobin, (MCHC) mean corpuscular hemoglobin concentration.

Table 4. Least square mean ( \pm standard error) of fibrinogen (Fibri), total leukocytes (Tot Leuk), segmented neutrophils (Seg Neut), lymphocytes (Lymph), eosinophils (Eosin), and monocytes (Mono) of Santa Ines ewes according to physiological stage.

\begin{tabular}{|c|c|c|c|c|c|c|c|}
\hline & & Fibri. & $\begin{array}{l}\text { Tot.Leuk. } \\
(/ \mu \mathrm{L})\end{array}$ & $\begin{array}{c}\text { Seg Neut } \\
(/ \mu \mathrm{L})\end{array}$ & $\begin{array}{l}\text { Lymph } \\
(/ \mu \mathrm{L})\end{array}$ & $\begin{array}{l}\text { Eosin } \\
(/ \mu L)\end{array}$ & $\begin{array}{l}\text { Mono } \\
(/ \mu \mathrm{L})\end{array}$ \\
\hline \multirow[t]{3}{*}{ Pregnancy } & $>30$ & $1.22 \pm 0.03$ & $9161 \pm 348^{\mathrm{a}}$ & $4883 \pm 2634^{\mathrm{a}}$ & $3580 \pm 841^{\mathrm{a}}$ & $373 \pm 65^{\mathrm{a}}$ & $491 \pm 27^{a}$ \\
\hline & $>14-30$ & $1.26 \pm 0.02$ & $8751 \pm 371^{\mathrm{a}}$ & $4854 \pm 2954^{\mathrm{a}}$ & $3021 \pm 938^{b}$ & $481 \pm 71 \mathrm{a}^{\mathrm{b}}$ & $332 \pm 30^{\mathrm{b}}$ \\
\hline & $>1-13$ & $1.25 \pm 0.02$ & $9385 \pm 374^{\mathrm{a}}$ & $6227 \pm 2954^{b}$ & $2325 \pm 938^{c}$ & $589 \pm 71^{b}$ & $193 \pm 30^{c}$ \\
\hline \multirow[t]{3}{*}{ Peripartum } & Dia 0 & $1.22 \pm 0.02$ & $10918 \pm 371^{\mathrm{b}}$ & $18466 \pm 3061^{\mathrm{c}}$ & $5860 \pm 972^{b}$ & $269 \pm 73^{c}$ & $68 \pm 31^{\mathrm{cd}}$ \\
\hline & $1-13$ & $1.24 \pm 0.02$ & $9480 \pm 374^{\mathrm{a}}$ & $6390 \pm 2954^{b}$ & $2388 \pm 938^{\mathrm{bcd}}$ & $527 \pm 71^{\mathrm{ab}}$ & $110 \pm 30^{\text {ce }}$ \\
\hline & $\geq 14-27$ & $1.25 \pm 0.02$ & $9404 \pm 371^{\mathrm{a}}$ & $6719 \pm 2989^{b}$ & $2199 \pm 949^{c}$ & $503 \pm 71^{\mathrm{ab}}$ & $96 \pm 31^{\mathrm{cf}}$ \\
\hline \multirow[t]{3}{*}{ Lactation } & $>28-55$ & $1.26 \pm 0.02$ & $8931 \pm 305^{\mathrm{a}}$ & $6341 \pm 2157^{b}$ & $2158 \pm 690^{\mathrm{c}}$ & $484 \pm 54^{\text {ad }}$ & $91 \pm 22^{\mathrm{def}}$ \\
\hline & $>56-83$ & $1.26 \pm 0.02$ & $9289 \pm 309^{\mathrm{a}}$ & $6183 \pm 2168^{b}$ & $2184 \pm 694^{c}$ & $666 \pm 55^{\text {bd }}$ & $101 \pm 22^{\text {def }}$ \\
\hline & $>84$ & $1.23 \pm 0.03$ & $9347 \pm 429^{\mathrm{a}}$ & $5544 \pm 3525^{\mathrm{b}}$ & $2723 \pm 1119^{\text {bd }}$ & $197 \pm 83^{c}$ & $133 \pm 36^{\text {cdef }}$ \\
\hline
\end{tabular}

Different superscript letters $(\mathrm{a}, \mathrm{b}, \mathrm{c}, \mathrm{d}, \mathrm{e})$ within the same column indicate significant differences at $\mathrm{p}<0.05$ (Tukey-Kramer). 
Table 5. Mean ( \pm standard error) of globulin, albumin, serum protein (Ser Pt), urea, creatinine (Creatinin), and glucose (Gluco) of Santa Ines ewes according to physiological stage.

\begin{tabular}{|c|c|c|c|c|c|c|c|}
\hline & & $\begin{array}{l}\text { Globulin } \\
\text { (g/dL) }\end{array}$ & $\begin{array}{l}\text { Albumin } \\
\text { (g/dL) }\end{array}$ & $\begin{array}{l}\text { Ser Pt. } \\
\text { (g/dL) }\end{array}$ & $\begin{array}{c}\text { Urea } \\
(\mathrm{mg} / \mathrm{dL})\end{array}$ & $\begin{array}{c}\text { Creatinin } \\
(\mathrm{mg} / \mathrm{dL})\end{array}$ & $\begin{array}{c}\text { Gluco } \\
\text { (mg/dL) }\end{array}$ \\
\hline \multirow[t]{3}{*}{ Pregnancy } & $>30$ & $4.06 \mathrm{a}^{\mathrm{b}}$ & $2.41^{\mathrm{ab}}$ & $6.41^{\mathrm{a}}$ & $40.09 \pm 1.85^{\mathrm{a}}$ & $1.01 \pm 0.024^{\mathrm{ab}}$ & $47.3 \pm 1.06^{\mathrm{a}}$ \\
\hline & $>14-30$ & $4.00^{\mathrm{b}}$ & $2.51^{\mathrm{a}}$ & $6.53^{\mathrm{ab}}$ & $39.79 \pm 2.19^{\mathrm{a}}$ & $1.05 \pm 0.027^{\mathrm{a}}$ & $46.3 \pm 1.23^{\mathrm{a}}$ \\
\hline & $>1-13$ & $4.04^{\mathrm{b}}$ & $2.49^{\mathrm{a}}$ & $6.51^{\mathrm{a}}$ & $28.54 \pm 2.23^{b}$ & $1.14 \pm 0.027^{\mathrm{c}}$ & $48.6 \pm 1.23^{\mathrm{ab}}$ \\
\hline \multirow[t]{3}{*}{ Peripartum } & Dia 0 & $3.90^{\mathrm{b}}$ & $2.09^{c}$ & $5.97^{\mathrm{c}}$ & $24.180 \pm 2.22^{\mathrm{bc}}$ & $1.13 \pm 0.027^{\mathrm{c}}$ & . \\
\hline & $1-13$ & $3.99^{\mathrm{b}}$ & $2.36^{\mathrm{bd}}$ & $6.29^{\mathrm{a}}$ & $21.65 \pm 2.24^{\mathrm{c}}$ & $0.99 \pm 0.027^{\mathrm{ad}}$ & $51.5 \pm 1.25^{\mathrm{bcd}}$ \\
\hline & $\geq 14-27$ & $3.88^{\mathrm{b}}$ & $2.43^{\mathrm{adf}}$ & $6.37^{\mathrm{a}}$ & $26.69 \pm 2.22^{\mathrm{bc}}$ & $0.90 \pm 0.027^{\mathrm{e}}$ & $58.6 \pm 1.23^{\mathrm{c}}$ \\
\hline \multirow[t]{4}{*}{ Lactation } & $>28-55$ & $4.29^{\mathrm{a}}$ & $2.47^{\mathrm{a}}$ & $6.76^{\text {bd }}$ & $33.16 \pm 1.63^{b}$ & $0.92 \pm 0.021^{\mathrm{ef}}$ & $55.0 \pm 0.95^{\mathrm{e}}$ \\
\hline & $>56-83$ & $4.60^{\mathrm{c}}$ & $2.34^{\text {bef }}$ & $6.94^{\mathrm{e}}$ & $49.57 \pm 1.64^{\mathrm{d}}$ & $0.97 \pm 0.022^{\text {bdf }}$ & $52.9 \pm 0.95^{\mathrm{de}}$ \\
\hline & $>84$ & $4.85^{\mathrm{d}}$ & $2.07^{\mathrm{c}}$ & $6.96^{\mathrm{de}}$ & $52.91 \pm 2.52^{\mathrm{d}}$ & $0.97 \pm 0.033^{\text {bde }}$ & $50.2 \pm 1.4^{\mathrm{bd}}$ \\
\hline & SE & \pm 0.1 & \pm 0.1 & \pm 0.1 & - & - & - \\
\hline
\end{tabular}

Different superscript letters (a, b, c, d, e ) within the same column indicate significant differences at $\mathrm{p}<0.05$ (Tukey-Kramer).

Table 6. Mean ( \pm standard error) of total bilirubin (BT), direct bilirubin (BD), indirect bilirubin (BI), cholesterol, and AST of Santa Ines ewes according to physiological stage.

\begin{tabular}{ccccccc}
\hline & & BT(mg/dL) & BD(mg/dL) & BI(mg/dL) & Cholesterol(mg/dL) & AST (U/L) \\
\hline Pregnancy & $>30$ & $0.240 \pm 0.03^{\mathrm{a}}$ & $0.094 \pm 0.01^{\mathrm{ac}}$ & $0.101 \pm 0.02^{\mathrm{a}}$ & $54.48 \pm 1.6^{\mathrm{ab}}$ & $83.61 \pm 3.5^{\mathrm{acd}}$ \\
& $>14-30$ & $0.181 \pm 0.03^{\mathrm{a}}$ & $0.068 \pm 0.01^{\mathrm{ad}}$ & $0.125 \pm 0.03 \mathrm{~b}^{\mathrm{c}}$ & $56.99 \pm 1.8^{\mathrm{ac}}$ & $97.47 \pm 3.7^{\mathrm{b}}$ \\
& $>1-13$ & $0.258 \pm 0.03^{\mathrm{a}}$ & $0.072 \pm 0.01^{\mathrm{ad}}$ & $0.193 \pm 0.03^{\mathrm{b}}$ & $60.82 \pm 1.8^{\mathrm{c}}$ & $95.13 \pm 3.7^{\mathrm{b}}$ \\
& & & & & \\
Peripartum & Dia 0 & $0.023 \pm 0.03^{\mathrm{b}}$ & $0.007 \pm 0.01^{\mathrm{b}}$ & $0.020 \pm 0.03^{\mathrm{d}}$ & $49.81 \pm 1.7^{\mathrm{de}}$ & $92.95 \pm 3.7^{\mathrm{b}}$ \\
& $1-13$ & $0.458 \pm 0.03^{\mathrm{c}}$ & $0.122 \pm 0.01^{\mathrm{c}}$ & $0.326 \pm 0.03^{\mathrm{e}}$ & $48.16 \pm 1.8^{\mathrm{df}}$ & $83.34 \pm 3.7^{\mathrm{cd}}$ \\
& $\geq 14-27$ & $0.364 \pm 0.03^{\mathrm{d}}$ & $0.074 \pm 0.01^{\mathrm{ad}}$ & $0.295 \pm 0.03^{\mathrm{e}}$ & $51.44 \pm 1.8^{\mathrm{bd}}$ & $82.57 \pm 3.7^{\mathrm{cd}}$ \\
& $>28-55$ & $0.249 \pm 0.02^{\mathrm{a}}$ & $0.083 \pm 0.01^{\mathrm{ad}}$ & $0.166 \pm 0.02^{\mathrm{cf}}$ & $52.63 \pm 1.4^{\mathrm{beg}}$ & $94.57 \pm 3.2^{\mathrm{b}}$ \\
& $>56-83$ & $0.254 \pm 0.02^{\mathrm{a}}$ & $0.076 \pm 0.01^{\mathrm{ad}}$ & $0.183 \pm 0.02^{\mathrm{bf}}$ & $55.28 \pm 1.4^{\mathrm{ag}}$ & $99.20 \pm 3.2^{\mathrm{ab}}$ \\
& $>84$ & $0.210 \pm 0.04^{\mathrm{a}}$ & $0.047 \pm 0.02^{\mathrm{bd}}$ & $0.173 \pm 0.03^{\mathrm{abf}}$ & $49.64 \pm 2.1^{\mathrm{bef}}$ & $111.0 \pm 4.3^{\mathrm{e}}$ \\
\hline
\end{tabular}

Different superscript letters $\left({ }^{\mathrm{a},} \mathrm{b}, \mathrm{c}, \mathrm{d}, \mathrm{e}\right)$ within the same column indicate significant differences at $\mathrm{p}<0.05$ (Tukey-Kramer). 
Table 7. Mean ( \pm standard error) of calcium, phosphorus, iron $(\mathrm{Fe})$ of Santa Ines ewes according to the physiological period.

\begin{tabular}{ccccc}
\hline & & $\begin{array}{c}\text { Calcium } \\
(\mathbf{m g} / \mathbf{d L})\end{array}$ & $\begin{array}{c}\text { Phosphorus } \\
(\mathbf{m g} / \mathbf{d L})\end{array}$ & $\begin{array}{c}\mathbf{F e} \\
(\boldsymbol{\mu g} / \mathbf{d L})\end{array}$ \\
\hline Pregnancy & $>30$ & $8.05 \pm 0.162 \mathrm{ab}$ & $5.29 \pm 0.22 \mathrm{a}$ & $197.53 \pm 7.04 \mathrm{abd}$ \\
& $>14-30$ & $8.43 \pm 0.194 \mathrm{a}$ & $4.72 \pm 0.24 \mathrm{~b}$ & $207.44 \pm 10.27 \mathrm{abd}$ \\
& $>1-13$ & $8.22 \pm 0.239 \mathrm{ab}$ & $4.72 \pm 0.24 \mathrm{~b}$ & $197.16 \pm 10.08 \mathrm{abd}$ \\
Peripartum & Dia 0 & $9.02 \pm 0.239 \mathrm{cde}$ & $4.93 \pm 0.24 \mathrm{ab}$ & $\cdot$ \\
& $1-13$ & $9.51 \pm 0.251 \mathrm{cf}$ & $5.29 \pm 0.24 \mathrm{a}$ & $183.35 \pm 9.71 \mathrm{abc}$ \\
& $\geq 14-27$ & $7.79 \pm 0.233 \mathrm{~b}$ & $5.02 \pm 0.24 \mathrm{ab}$ & $220.28 \pm 10.07 \mathrm{~d}$ \\
& $>28-55$ & $9.03 \pm 0.144 \mathrm{cg}$ & $4.75 \pm 0.20 \mathrm{~b}$ & $185.37 \pm 6.80 \mathrm{bc}$ \\
& $>56-83$ & $9.38 \pm 0.150 \mathrm{dfg}$ & $5.05 \pm 0.20 \mathrm{ab}$ & $168.17 \pm 6.67 \mathrm{c}$ \\
& $>84$ & $8.46 \pm 0.265 \mathrm{ae}$ & $4.98 \pm 0.28 \mathrm{ab}$ & $175.92 \pm 9.64 \mathrm{c}$ \\
\hline
\end{tabular}

Different superscript letters $(\mathrm{a}, \mathrm{b}, \mathrm{c}, \mathrm{d}, \mathrm{e})$ within the same column indicate significant differences at $\mathrm{p}<0.05$ (Tukey-Kramer).

\section{Discussion}

Previous research suggests that feed intake of sheePrevious research suggests that feed intake of sheep and other ruminants is often insufficient in the final period of gestation and early lactation. Our results show that although a decrease in dry matter and crude protein intake occurred in these stages, consumption was sufficient to meet the animals' requirements in these physiological states (NRC, 2006) in confined conditions. Accordingly, we observed relatively small weight and body condition score variations.

Although the peripartum period is often associated with increased susceptibility to gastrointestinal parasitic infections, there were no significant variations in trichostrongylid counts between gestational and peripartum periods. Haemonchus spp. genus are highly pathogenic blood-suckers, causing a decrease in the packed cell volume and hence increasing the ocular mucosa coloration score (FAMACHA). Haemonchus spp. had high mean prevalence (92.85\%), while Trichostrongylid egg counts decreased during the experiment. The FAMACHA score was constant during different physiological conditions. It is unlikely that this low association between Trichostrongylid
FEC and the FAMACHA score resulted from a subjective assignment of this score since the association between PCV and FAMACHA was high. Therefore, the weak association between FAMACHA score and Trichostrongylid count may have resulted from the fact that sheep were already infected before the experiment. Although the Trichostrongylid FEC was high until the peripartum period and decreased with the onset of lactation, the appropriate protein intake might have allowed maintenance of PCV values predominantly within the range considered normal for sheep (27 to $45 \%$; Pugh, 2005) throughout the experiment. The only exception was a small decrease in PCV just after the beginning of lactation, but was followed by swift recovery to values within the normal range. Other parasites with lower pathogenicity than Haemonchus spp. (Afonso et al., 2013) were also identified, such as Trichostrongyloidea (Trichostrongylus, Cooperia and Oesophagostomum), Strongyloides spp., Eimeria spp. and Moniezia spp. Moniezia and Eimeria FEC were higher in the pregnancy and lactation periods, respectively, probably due to the management used. Better food, confined animals are less exposed to infection by Moniezia (albeit more exposed to infection by Eimeria due to trough feeding). Although Haemonchus spp. can cause severe anemia and 
hypoproteinemia (Faria Junior et al., 2002), depression, loss of body condition, reduced productivity, and even death (Kaplan et al., 2004), no animal died or had to be dewormed before or during our experiment.

As expected, there was a variation in the hematological profile of animals between periods. The values of hematological variables were predominantly below the normal range for the species, possibly due to the negative action that hormone variation has on hematological variables (Kerr, 2003). Hematological parameters change easily and fast during peripartum (Cardoso et al., 2010) and the PCV decreases as pregnancy progresses due to the physiological hemodilution in this period (Brito et al., 2006). Recent studies suggest that even healthy Santa Ines sheep have lower erythrocyte, hemoglobin and PCV levels and higher MCHC values (Salviano et al., 2013) compared to the reference values for the species found in the literature (Schalm, 1981; Harvey and Meyer, 1998; Pugh, 2005). Our results showed increased amounts of red blood cells and hemoglobin from pregnancy until the end of lactation. However, we did not observe lower PCV values during gestation, as reported in the literature (Brito et al., 2006). We also found higher MCHC values in relation to the literature, including those suggested by Salviano et al. (2013) for nonpregnant sheep. These findings reinforce the hypothesis that breed can significantly affect hematological patterns, and emphasizes the need for studies on specific breeds considering age, sex and physiological condition to avoid misinterpretation of results.

During pregnancy, hormone action on the immune system (Kerr, 2003) is necessary to suppress the defense mechanisms and thus promote survival and development of the fetus (Lloyd, 1983). The low total leukocyte levels observed before delivery may be related to this factor, while the high level on the day of delivery may be related to the migration of these cells into the uterus, where there is tissue injury caused by birth.
Segmented neutrophils act as a first line of defense against infection. Many researchers have investigated the activity of these leukocytes close to delivery (Guidry et al., 1976; Fonteque et al., 2013). The level of segmented neutrophils increased as delivery approached, peaking on day 0 (delivery), with a gradual decrease afterward. This subsequent reduction may be related to the migration of these cells to injury sites, corroborating the results of Fontequete et al. (2013), who observed decrease in total leukocytes and segmented neutrophils during postpartum. However, they also noted increased segmented neutrophils and total leukocytes after delivery, indicating the likely need to replenish the number of circulatory cells due to their flow to the uterus. We did not observe this pattern, probably because sheep were parasitized, which kept the immune system active.

The observed decrease in lymphocytes and monocytes before delivery, starting 30 days beforehand and declining sharply 15 days before delivery, may have occurred because of migration of cells to the uterus and or abomasum. Migration of leukocytes to the abomasum occurs to enable antigen recognition of the nematode, followed by stimulation of the immune system to generate the appropriate response to fight parasites (Douch, 1990). The activation of larvae, previously in hypobiosis, happens due to immunosuppression in response to circulating cortisol in the days before delivery. The number of EPG also increased in these same periods. Lymphocyte concentration was lower during postpartum than on the delivery day, whereas monocytes showed higher values 15 days after delivery and after more than 84 days of lactation. The high score in the first period after delivery may be related to peak lactation and attempt of the body to restore immunity after the stress period caused by constant hormonal changes.

Eosinophil counts increased gradually until the day of delivery, decreased considerably in the delivery day, and then rose gradually in the postpartum period until 84 days in milk, after which the concentrations declined. These results are similar to those reported by Fonteque 
et al. (2013) who found that stress caused by birthing in goats is overcome in the first week after delivery, with more stable counts, a fact confirmed in our study. However, these findings contrast with those of Sabag et al. (1978) who noted a decreasing number of circulating eosinophils due to the action of cortisol associated with inhibition of eosinophilopoiesis in the marrow and/or migration of these cells to other tissues.

The globulin profile behaved proportionally to the nutritional requirement in each physiological condition, with a progressive increase from the peak of lactation $(\geq 14$ 27 days) until the end of lactation, when the demand decreased due to lower milk production. Moreover, our results showed a decrease of serum albumin and protein values in the peripartum period, specifically on the day of birth, with subsequent increase during lactation. This decline can be expected to occur starting in the last third of gestation until peak lactation, due to three main factors. First, there is a decrease in consumption of dry matter and crude protein in late pregnancy and early lactation. Second, there is a larger requirement of the fetus in this stage, with $70 \%$ of growth happening in the last 50 days of pregnancy (Russell, 1979). Finally, there is higher nutritional requirement until peak milk production (Houdijk, 2008), with changes in hormone concentrations to promote gluconeogenesis and mobilization of adipose tissue to provide energy to the fetus and the mammary glands (Rodrigues et al. 2007). However, the decrease in serum albumin and protein levels occurred only on the day of delivery, although food consumption (DM and CP) apparently met the animals' requirements, as demonstrated by maintenance of body condition throughout the experiment. The specific reasons for the reduction in these parameters only on the lambing day remain to be determined.

The urea profile is directly related to protein intake and renal function; changes in its level indicate possible kidney disorders.
As expected, THE UREA VALUES FOUND IN THIS STUDY FOLLOWED the protein intake pattern in the different physiological stages, with decrease from gestation to peripartum periods and subsequent increase in the lactation period. Variations in creatinine were not related to these periods and were always below the normal range for the species (1.2 to $1.7 \mathrm{mg} / \mathrm{dL})$.

Calcium values increased from the gestation peripartum. There was a decrease in the level of this mineral during peak milk production ( $\geq 14-27)$ in relation to peripartum, which might be related to milk production requirements. However, endocrine control of calcium means calcium is not a good nutritional indicator, although this mineral is closely associated with metabolism in the peripartum period (Gonzalez, 2000). The small variation in serum phosphorus profile was not related to the physiological condition. However, in some stages of gestation, peripartum and lactation, values lower than the normal ones for other sheep breeds were noted ( 5.1 to $7.3 \mathrm{mg} / \mathrm{dL}$, Gonzalez et al., 2006). In the postpartum period, this mineral -which is an indirect indicator of energy metabolism- can be used in the form of adenosine triphosphate, which would cause a decrease in its concentration (Feijó et al., 2014).

According to Borjesson et al. (2000), glucose concentration in ruminants is readily altered with stress and excitement due to catecholamine release. Since the assessed periods can be considered stressful, especially peripartum, the changes noted can be considered normal. However, ruminants have an excellent mechanism for maintenance of glucose, keeping it at relatively constant concentrations. This makes observations of large changes unlikely, because only very severe dietary restrictions would lead to hypoglycemia (Peixoto et al., 2010).

An increase in AST levels is associated with liver and/or muscle damage. However, our results were within the normal range for the species and in accordance with those reported by Avellanet et al. (2007) for the Xisqueta breed (66.0 to 194.0 U/L). Similarly, 
increased concentration of blood bilirubin is most commonly caused by hemolysis or liver diseases (Raposo et al., 2004). Batista et al. (2009), studying healthy sheep in two age groups (3-5 years and over 5 years), found mean values of total bilirubin of 0.046 and $0.037 \mathrm{mg} /$ $\mathrm{dL}$, direct bilirubin of 0.020 and $0.016 \mathrm{mg} / \mathrm{dL}$, and indirect bilirubin of 0.026 and $0.021 \mathrm{mg} /$ $\mathrm{dL}$, respectively. However, according to Pugh (2005), normal values for the total and direct bilirubin are between 0.1 to $0.5 \mathrm{mg} / \mathrm{dL}$ and 0.0 to $0.27 \mathrm{mg} / \mathrm{dL}$, respectively. Thus, despite the variation found in different physiological periods, just total bilirubin on the delivery day had a lower average than what is considered normal for the species.

There was little variation in cholesterol levels throughout the experiment, with lower values in the peripartum and at the end of lactation -when the animals were already at a lower nutritional level to promote drying of milk. Ribeiro et al. (2004) found no difference in cholesterol levels between empty and pregnant ewes and suggested that blood metabolites do not vary with the reproductive status of well-fed sheep. However, Peixoto et al. (2010) reported that formation of steroid hormones during gestation causes part of the cholesterol in the blood to be reabsorbed, decreasing its level at that stage.

Mendonça et al. (2005) reported that serum changes of some minerals, including iron, can result from infectious diseases of ruminants, including mastitis. Kaneko et al. (1997) considered serum iron values between 166 and $221 \mathrm{mg} / \mathrm{dL}$ to be normal for the species. Despite the variations in periods, the concentrations remained within the normal range.

The results of this study indicate that the peripartum period exerts an influence on the hematological, parasitological and biochemical parameters of sheep. Nonetheless, correct knowledge and interpretation of physiological parameters during this phase can prevent mistaken diagnosis and provide adequate support during this stage.

\section{Declarations}

\section{Funding}

This study was financed in part by the Coordenação de Aperfeiçoamento de Pessoal de Nível Superior - Brasil (CAPES) (Finance Code 001), and by the Foundation for Research Support of the State of São Paulo (Proc. Fapesp 2009-54564-7).

\section{Conflicts of interest}

The authors declare they have no conflicts of interest with regard to the work presented in this report.

\section{Author contributions}

Caroline M G David - collected the data and wrote the manuscript.

Ricardo L D Costa - conception of the study; administered the project and reviewed the manuscript.

Guadalupe A E Parren - collected the data.

Miguel A S Rua - collected the data.

Ellen C P Nordi - collected the data.

Cláudia C P Paz - statistical analysis.

Celia R Quirino - critical Reading of the manuscript.

Rogério S Figueiredo - collected the data and conducted laboratory analysis.

Elizabeth Bohland - laboratory analysis.

\section{References}

Afonso VAC, Costa RLD, Soares-FilhoCV, Cunha EA, Perri SHV, Bonello FL. Supplementation with protected fat to manage gastro-intestinal nematode infections in Santa Inês sheep. Semin Cienc Agrar 2013; 34:1227-1238. DOI: https:/doi.org/10.5433/1679-0359.2013v34n3p1227-1238 
Amarante AFT, Bricarello PA, Rocha RA, Gennari SM. Resistance of Santa Inês, Suffolk and Ile de France sheep to naturally acquired gastrointestinal nematode infections. Vet Parasitol 2004; 120:91-106. DOI:https://doi.org/10.1016/j.vetpar.2003.12.004

Avellanet R, Cuenca R, Pastor J, Jordana J. Parámetros hematológicos y bioquímico clínicos en la raza ovina xisqueta. Archiv Zootec 2007; 56:498-501. DOI: https://www.redalyc.org/pdf/495/49509922.pdf

Aya S, Neves EP, Castilho MFO, Mexia AA. Infecção helmíntica em ovelhas Santa Inês no periparto criadas na região do Pantanal Brasileiro. Rev Bras Saude Prod Ani 2008; 9(2):321-326. DOI: http://revistas.ufba.br/index.php/rbspa/article/view/889/629

Batista MCS, Castro RS, Rego EW, Carvalho FAA, Silva SMMS, Carvalho CCD, Correa FR. Hemograma, proteinograma, ionograma e dosagens bioquímicas e enzimáticas de ovinos acometidospor conidiobolomicose no Nordeste do Brasil. Pesq Vet Bras 2009; 29 (1):17-24. DOI: http://www.scielo.br/pdf/pvb/v29n1/a02v29n1.pdf

Borjesson DL, Christopher MM, Boyce WM. Biochemical and hematologic reference intervals for free-ranging desert bighorn sheep. J Wildl Dis 2000; 36 (2):294-300. DOI: http://dx.doi.org/10.7589/0090-3558-36.2.294

Brito MA, Gonzalez FD, Ribeiro LA, Campos R, Barbosa PR, Bergman G. Composição do sangue e do leite em ovinos leiteiros do sul do Brasil: variações na gestação e lactação. Cienc Rural 2006; 36:1-7. DOI: http://x.doi.org/10.1590/S0103-84782006000300033

Cardoso EC, Oliveira DR, Dourado AP, Araujo CV, Ortalani EL, Brandão FZ. Peso e condição corporal, contagem de OPG e perfil metabólico sanguíneo de ovelhas da raça Santa Inês no periparto, criadas na região da Baixada Litorânea do Estado do Rio de Janeiro. Rev Bras Cienc Vet 2010; 17:77-82. DOI: http://periodicos.uff.br/rbcv/article/view/6992/5274
Douch PGC. The sheeps imune response to nematode parasites and prospects for its exploitation. In: Proceedings of the new zealand society of animal production. Proceedings 1990; 50:229-236.

Donaldson J, Van-Hourten MJF, Sykes AR. The effect of dietary fishmeal supplementation on parasite burdens of periparturient sheep. Anim Sci 2001; 72:199-205. DOI: https://doi.org/10.1017/S1357729800055648

Donaldson J, Van-Hourten MJF, Sykes AR. The effect of nutrition on the periparturient parasite status of mature ewes. Anim Sci 1998; 67:523-533. DOI: https://doi.org/10.1017/S1357729800032951

Faria JR SP, Silva MM, Scheibel M, Martins MF, Rabello P, Bertagnon HG, Garcia M. Uso da contagem fecal de ovos de nematóides (OPG) para estimar a condição clínica em caprinos. Cienc Vet Trop 2002; 5:86-92. http://www.rcvt. org.br/volumes/volume_5, numero_2_3, maio dezembro,2002.pdf

Feijó JO, Aragão RB, Ferreira MB, Perazzoli D, Rabassa VR, Martins CF, Del-Pino FAB, Silva LGC, Corrêa MN, Pereira RA. Avaliação de parâmetros bioquímicos clínicos de ovelhas dogrupo genético pantaneiro gestantes e não gestantes. Braz J Vet Res Anim Sci 2014; 51, 2:111-117. DOI: https://doi.org/10.11606/issn.1678-4456.v51i2p111-117

Fonteque JH, Saito ME, Barioni G, Valente ACS, Takahira RK, Kohayagawa A. Leucograma e metabolismo oxidativo de neutrófilos em cabras da raça Saanen nos períodos de gestação, parto e pós-parto. Pesq Vet Bras 2013;33(1):63-70. DOI: http://dx.doi.org/10.1590/S0100-736X2013001300011

Gennari SM, Blasques LS, Rodrigues, AAR, Cilento MC, Souza, SLP, Ferreira, F. Determinação da contagem de ovos de nematódeos no período peri-parto em vacas. Braz J Vet Res Anim Sci. 2002; 39(1):32-37. DOI: http://dx.doi.org/10.1590/S1413-95962002000100006 
Gonzaléz FHD, Barcellos J, Patiño HO, Ribeiro LA. Perfil metabólico de ruminantes, seu uso em nutrição e doenças nutricionais. Biblioteca setorial da faculdade de medicina veterinária UFRGS. 2010; 108p. https://lume.ufrgs.br/ bitstream/handle/10183/26657/000274557. pdf? sequence $=1$ \&isAllowed $=y$

Gonzaléz FHD, Silva SC. Introdução à clínica veterinária.PortoAlegre:GráficadaUniversidade Federal do Rio Grande do Sul. 2006; 357p. http:/www.ufrgs.br/bioquimica/arquivos/ibcv.pdf

GordonHMCL, WhitlockHV.Anew techniquefor counting nematode eggs in sheep faeces. J Counc Sci Ind Res 1939; 12:50-52. https://publications. csiro.au/rpr/download?pid=procite:21259a33$\underline{\text { 8a8e-4add-9315-f8338091a3e6\&dsid=DS1 }}$

Guidry AJ, Paape MJ, Pearson RE. Effects of parturition and lac $\neg$ tation on blood and milk cell concentrations, corticosteroids, and neu $\neg$ trophil phagocytiosis in the cow. Anim J Vet Res 1976; 37(10):1195-1200. https://www.ncbi.nlm.nih.gov/pubmed/984546

Hernandez PM, Salem AZM, Elghandour Hernandez PM, Salem AZM, Elghandour MMMY, Salazar MC, Lagunas BC, Camacho LM. Anthelmintic effects of Salix Babylonica L. and Leucaena leucacephala Lam. extracts in growing lambs. Trop Anim Health Prod 2013; 46:173-178. DOI: https://doi.org/10.1007/s11250-013-0471-7

Houdijk JGM. Influence of periparturient nutritional demand on resistance to parasites in livestock. Parasite Immunol 2008; 30:113-121. https://doi.org/10.1111/j.13653024.2008.00992.x

Houdijk JGM, Kyriazakis I, Jackson F, Coop RL. The expression of immunity toTeladorsagia circumcincta and its relationship to protein nutrition depend on body protein reserves. Parasitol 2001; 122:661-672. DOI: https://doi.org/10.1017/S0031182001007922
Hoste H, Torres-Acosta JF, Paolini V, Aguilar-Caballero A, Etter E, Lefrileux Y, Chartier $\mathrm{C}$, Broqua $\mathrm{C}$. Interactions between nutrition and gastrointestinal infections with parasitic nematodes in goats. Small Rumin Res 2005; 60:141-151. DOI: https://doi.org/10.1016/j.smallrumres.2005.06.008

Kaneko JJ, Harvey JW, BRUSS ML. Clinical biochemistry of domestic animals. 5th ed. New York; Academic Press. 1997. https://www.sciencedirect.com/book/9780123704917/ clinical-biochemistry-of-domestic-animals

Kaplan RM, Burke JM, Terrill TH, Miller E, Getz WR, Mobini S, Valencia E, Williams MJ, Williamson LH, Larsen M, Vatta AF. Influence of dietary protein supply on resistance to experimental infections with Haemonchus contortus in Ile de France and Santa Ines lambs. Vet Parasitol 2005; 134:99-109. DOI: https://doi.org/10.1016/j.vetpar.2005.05.068

Kerr MG. Exames Laboratoriais em Medicina Veterinária, Bioquímica Clínica e Hematologia. 2 ed. São Paulo: Roca. 2003; 425p.

Mapa. Temas: Caprinos e Ovinos. Ministério da Agricultura, Pecuária e Abastecimento, 2013. Access date: November, 2014. URL http://www.agricultura.gov.br/assuntos/ camaras -s etoriais-tematicas / camarassetoriais-1/caprinos-e-ovinos

Mendonça, CL, Afonso, JAB, Costa, NA. Mastite em Ovelhas. Vet. Zootec. 2005; 25, 7.

Meyer, DJ, Harvey, JW, (2. ed.). Veterinary laboratory medicine: interpretation and diagnosis. Philadelphia: W.B. Saunders Company. 1998; 373p. DOI: https://doi.org/10.1111/j.1939-165X.2004.tb00372.x

NationalResearchConcil-NRC.,2006.Nutrient requirements of small ruminants. Washington, D.C.: National Academy Press. 1998. https://www.nap.edu/read/11654/chapter/1 
Nieto LM, Martins EN, Macedo FAF, Zundt M. Observações epidemiológicas de helmintos gastrintestinais em ovelhas mestiças manejadas em pastagens com diferentes hábitos de crescimento. Cienc. Anim. 2003; 4(1):45-51. https://www.revistas.ufg.br/vet/article/view/297

Peixoto LAO, Osório MTM, Osório JCS, Nörnberg JL, Pazini M. Desempenho reprodutivo e metabólitos sanguíneos de ovelhas Ile de France sob suplementação com sal orgânico ou sal comum durante a estação de monta. Rev Bras Zootec 2010; 39 (1):191-197. DOI: http://dx.doi.org/10.1590/S1516-35982010000100025

Pugh DG. Clínica de ovinos e caprinos. São Paulo: Roca. 2005; 513p.

Pugh DG. Clínica de Ovinos e Caprinos. Roca, São Paulo. 2004.

Raposo JB, Fernandes CG, Baialardi C, Driemeier D. Observações clínicas e bioquímicas em ovinos e bovinos intoxicados experimentamente por Myoporum laetum. Acta Sci Vet 2004; 32 (1):9-17. https://www.lume.ufrgs.br/handle/10183/19906

Ribeiro LAO, Mattos RC, González FHD, Wald VB, Silva MA, Rosa VL. Perfil metabólico de ovelhas Border Leicester $x$ Texel durante a gestacão e a lactação. Rev Portug Cienc Vet 2004; 99:155-159. http://www.fmv.ulisboa.pt/spcv/PDF/ pdf9 2004/551_155_159.pdf

Roberts FHS, O'Sullivan SP. Methods for egg counts and larval cultures for strongyles ingesting the gastrointestinal tract of cattle. Aust J Agric Res 1950; 1:99-102. DOI: https://doi.org/10.1071/AR9500099

Rocha RA, Amarante AFT, Bricarello PA. Comparison of the susceptibility of Santa Inês and Ile de France ewes to nematode parasitism around parturition and during lactation. Small Rumin Res 2004; 55:65-75. DOI: https://doi.org/10.1016/j.smallrumres.2003.12.004
Rodrigues CAF, Rodrigues MT, Branco RH, Carvalho GR, Torres RA, Torres-Filho RA. Avaliação do consumo e de metabólitos plasmáticos de cabras gestantes com duas condições corporais alimentadas com dietas formuladas com diferentes níveis de energia. Rev Bras Zootec 2007; 36:945-952. DOI: http://dx.doi.org/10.1590/S1516-35982007000400026

Rodrigues CAF, Rodrigues MT, Branco $\mathrm{RH}$, Queiroz AC, Araújo CV. Influência da condição corporal e da concentração de energia nas dietas no periparto sobre o desempenho de cabras em lactação. Revis Bras Zootec 2006; 35 (4):1560-1567. DOI: http://dx.doi.org/10.1590/S1516-35982006000500039

Russel AJF. The nutrition of the pregnant ewe. In: British Council. The management and diseases of sheep. Edinburg. 1979.

Salviano MB, Sousa Júnior A, Moura WL. Valores hematológicos de ovelhas Santa Inês adultas não prenhes. Rev Cient Eletron Med Vet 2013; http://faef.revista. inf.br/imagens_arquivos/arquivos destaque/ fCsRfEw1qA0b5gz_2013-6-19-17-3-33.pdf

Sanudo C, Sierra I. Calidad de la carnal em la especie ovina, Ovino 1986; 1:127-153.

SAS Institute. User's Guide to Statistics. Version 9.3. North Carolina, State University, Cary. 2011.

Schalm OW, Carrol EJ. Veterinary Hematology. Lea \& Febiger: Philadelphia. 1986.

Silva RMC, Quirino CR, Costa RLD, Neves GD, Santos CP, Albernaz AP, Silveira GR, Reis NC, Machado JA, Junior OAM. Efeito da variação mensal, propriedade e estado fisiológico sobre a infecção gastrintestinal por nematóides em ovelhas Santa Inês; Bol Indus Anim 2009; 66 (1):01-10. http://www.iz.sp.gov.br/bia/index.php/bia/ article/view/1105/1099 